\title{
The methods for searching hypervelocity star candidates from the SDSS
}

\author{
Yinbi Li, Ali Luo, Gang Zhao and Youjun Lu
}

Key Laboratory of Optical Astronomy, National Astronomical Observatories, Chinese Academy of Sciences, 20A Datun Road, 100012, Beijing, China email: ybli@bao.ac.cn

\begin{abstract}
Hyper-velocity stars are believed to be ejected out from the Galactic center through dynamical interactions of (binary) stars with the central massive black hole(s). In this paper, we firstly select F and G type main sequence stars from about 370,000 stellar spectra of DR7. Then, we select 369 high velocity stars from main sequence samples using the radial velocity distribution. Finally, we find 13 possible unbound hyper-velocity star candidates from the 369 high velocity stars.
\end{abstract}

Keywords. Hyper-velocity stars, SDSS, Origin

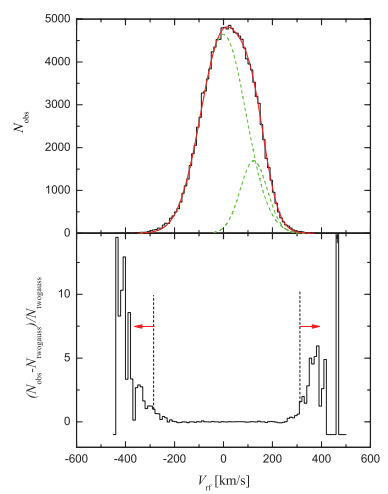

Figure 1. The top panel shows distribution of radial velocities in the Galactic rest-frame of $130775 \mathrm{~F}$ and G type stars, the best fit two Gaussian function (red curve) and the two Gauss components (dashed green curves). The bottom panel is the normalized residuals of the observations from the two Gaussian function. The two red arrows show the region of 369 high velocity stars.

Recently, more than 16 HVSs have been reported in the literature, and most of them are 3-4 $\mathrm{M}_{\odot}$ late B-type stars. Assuming a Salpeter initial mass function, the expected solar mass HVSs are about 10 times more abundant than 3-4 $\mathrm{M}_{\odot}$ HVSs. In this paper, we aim to search for low mass F and G type hyper-velocity star candidates from about 370,000 stellar spectra of the SDSS, and we search such HVS candidates with the following methods. (1) Select F and G type main sequence star samples with photometric and spectroscopic methods mentioned in Ivezić et al. (2008), and calculate their heliocentric distances. (2) Select high velocity stars from the Galactic radial velocity distribution of main sequence samples with normalized residuals larger than 1 which is shown in Figure 1, and the region of high velocity stars is marketed by two red arrows. (3) Select unbound HVS candidates from the high velocity stars through comparing their space total velocities and escape velocities. Finally, we find 13 HVS candidates from these 369 high velocity stars.

\section{References}

Ivezić, Ž., Sesar, Branimir, Jurić, Mario, et al 2008, ApJ, 684, 287 